\title{
Classification of Fallacies of Relevance ${ }^{1}$
}

\author{
Douglas Walton University of Winnipeg
}

\begin{abstract}
Fallacies of relevance, a major category of informal fallacies, include two that could be called pure fallacies of relevance-the wrong conclusion (ignoratio elenchi, wrong conclusion, missing the point) fallacy and the red herring (digression, diversion) fallacy. The problem is how to classify examples of these fallacies so that they clearly fall into the one category or the other, on some rational system of classification. In this paper, the argument diagramming software system, Araucaria, is used to analyze the argumentation in some selected textbook examples of pure fallacies of relevance. A system of classification of these fallacies is proposed, and criteria for determining whether an example should be classified as wrong conclusion or red herring are formulated. A key difference cited is that in a case where the red herring fallacy has been committed, even if the argument may go to a wrong conclusion, there is evidence of the use of a deceptive tactic of diversion. Textual evidence must indicate that the arguer deliberately interjects a distracting controversy to lead the respondent away from the real issue to be disputed.
\end{abstract}

Résumé: Les sophismes qui enfreignent la pertinence constituent une classe importante des sophismes non formels. Les sophismes de conclusion erronée (ignoratio elenchi) et de digression (ou de diversion) transgressent si évidemment la pertinence qu'on pourrait les nommer des purs sophismes de pertinence. Le défi est de déterminer comment classer des exemples dans l'une ou l'autre de ces catégories. J'emploie le logiciel Araucaria pour représenter en diagramme la structure d'exemples de purs sophismes qu'on trouve dans certains manuels, et propose des critères pour relever ce défi. Une différence principale entre ces deux sophismes est que pour imputer le sophisme de digression à une personne il faut des preuves qu'elle a délibérément employé des tactiques trompeuses ou des controverses pour dérouter son auditoire du vrai sujet en question.

Keywords: Red herring, wrong conclusion, missing the point, ignoratio elenchi, Araucaria, misdirected argumentation, diversionary tactics, chaining of argumentation, argument diagramming, ultimate probandum.

\section{Introduction}

This study moves the project of fallacy classification forward by using a new computerized method of argument diagramming called Araucaria (Reed and Rowe, 2002 ) to classify fallacies of relevance by analyzing and comparing paradigm examples. ${ }^{2}$ The two leading introductory logic textbooks, Copi and Cohen's Introduction to Logic and Hurley's A Concise Introduction to Logic, tend to lead 
the way by offering examples of these fallacies that have become familiar to generations of instructors and students. Such examples become the paradigms defining what failures of correct argumentation the fallacies in question are taken to commit. As these texts have gone through many editions, they have changed their paradigm examples, even though many of the earlier ones persist in fallacy lore through their use in other texts. ${ }^{3}$ As always with the study of fallacies, paradigm examples, while centrally important to define and explain the fallacy, can be difficult to classify. The commonest kind of classification problem arises from borderline cases that seem to straddle two different fallacies, or two subspecies of one type of fallacy. In this study, the two fallacies investigated are wrong conclusion (missing the point) and red herring, two pure fallacies of relevance.

The first one, well defined by Aristotle, is the fallacy of presenting an argument that may be valid, and have premises that are supported by evidence, but goes to the wrong conclusion. This mistake is a clear type of fallacy defined precisely by the Aristotelian analysis. The second, commonly known as red herring, is the fallacy of switching the topic of the discussion to some different issue that may be highly entertaining and diverting to the audience, distracting its attention from the real issue to be discussed. The problem posed is whether clear criteria can be devised that enable a critic to determine whether a given example falls under one category or the other. For in several key examples presented in the textbooks meant as paradigm instances of the second fallacy, the given argumentation can be analyzed in such a way that it goes to some conclusion other than the one that is supposed to be proved. To solve this problem of classification, and some other ones that are discussed as well, criteria for distinguishing between the two fallacies are presented, and two sets of guidelines for applying the criteria to real cases are formulated. It is argued that these criteria and guidelines can be used to develop a new system for classifying fallacies of relevance. However, as the reader will see clearly demonstrated by their analyses in this study, the examples treated turned out to be a lot more difficult to rationally classify than the authors or users of the textbooks might have imagined.

\section{Wrong Conclusion and Missing the Point}

According to the theory presented in (Walton, 2004), an argument is seen as a set of premises that is supposed to be used in a chain of argumentation to prove a designated proposition called its conclusion. The chain of argumentation is represented by an argument diagram is produced by filling in missing premises and conclusions as links in the chain. The designated conclusion, often called the ultimate probandum in law, is the proposition that is supposed to be proved. ${ }^{4}$ The simplest type of fallacy of relevance occurs in cases where the chain of argumentation starting from the premises proves the wrong conclusion, i.e., a proposition that may look like the conclusion to be proved, but is a different conclusion. This is the fallacy of ignoratio elenchi, or ignorance of refutation, described by Aristo- 
tle. ${ }^{5}$ Whatley (1870) presented quite a long and interesting list of examples of this type of fallacy. One is enough to cite here, also cited in (Walton, 2004, p. 53) along with four of Whately's other examples.

\section{The Relief of the Poor Example}

Instead of proving that "the poor ought to be relieved in this way rather than that," you prove that "the poor ought to be relieved" (Whately's italics).

Here, Whatley has analyzed the argumentation in the case by citing not only the conclusion supposed to be proved, but also the one that was actually proved instead. Thus on his analysis the fallacy committed in the case is that of wrong conclusion.

In Hurley's popular textbook (2003, p. 114), the category of fallacies of relevance is said to comprise those arguments where the conclusion seems to follow (psychologically) from the premises, but it does not follow (logically). Copi and Cohen (1994, p. 116) also classified this group of fallacies as characterized by the problem of confusing logical and psychological relevance. Hurley $(1994 ; 2003)$ classified eight fallacies as falling under the category of fallacies of relevance, including such fallacies as ad hominem, ad populum, ad baculum and ad misericordiam. Copi and Cohen (1994) classified twelve fallacies under this same category, including these four. In addition to these special fallacies of relevance, both books include a more general category, which could be called the pure fallacy of irrelevance. There has been a long tradition, stemming from Aristotle, of singling out one special fallacy, typically called ignoratio elenchi (ignorance of refutation), representing pure failure of relevance in an argument. Copi and Cohen (1994, p. 131) called it ignoratio elenchi or irrelevant conclusion, and held that it is committed when "an argument purporting to establish a particular conclusion is instead directed to proving a different conclusion". Hurley divided this category into two special subtypes called the missing-the-point fallacy and the red herring fallacy. Copi and Cohen (2004, p. 51) classified red herring as a subspecies of irrelevant conclusion.

The following example (Copi, 1982, p. 110) is a classic case of ignoratio elenchi.

\section{The Horrible Crime of Murder Example}

In a law court, in attempting to prove that the accused is guilty of murder, the prosecution may argue at length that murder is a horrible crime. He may even succeed in proving that conclusion. But when he infers from his remarks about the horribleness of murder that the defendant is 


\section{Douglas Walton}

guilty of it, he is committing the fallacy of ignoratio elenchi.

This example is a legal one. In law, for example in a criminal trial, there is a designated proposition that is supposed to be proved in a particular case, like "The defendant is guilty of murder". The judge has the duty to see that only relevant evidence is used in the argumentation presented by both sides, for a lawyer may wander away from proving what is supposed to be proved, for example by using emotional appeals to influence the jury. ${ }^{6}$ Copi's remarks, however, suggest a specific kind of failure of relevant argumentation when he remarked that such a line of argumentation may even succeed in proving the wrong conclusion, i.e., one that is different from the one to be proved.

On Hurley's account, the fallacy of missing the point (ignoratio elenchi) is said to occur where "the premises of an argument support one particular conclusion, but then a different conclusion, often vaguely related to the correct conclusion, is drawn" $(1994$, p. $125 ; 2003$, p. 123). This fallacy appears to be that of wrong conclusion. But the examples offered by Hurley suggest something that is perhaps slightly different. The following two examples are quoted from Hurley (1994, p. 126; 2003, p. 123).

\section{The Death Penalty Example}

Crimes of theft and robbery have been increasing at an alarming rate lately.

The conclusion is obvious: we must reinstate the death penalty immediately.

\section{The Welfare System Example}

Abuse of the welfare system is rampant nowadays.

Our only alternative is to abolish the system altogether.

According to Hurley's analysis (p. 123) these two arguments commit the fallacy of missing the point because they leap to a wrong conclusion, failing to even consider less radical solutions to the problem. The first argument leaps to the death penalty conclusion without even considering more moderate solutions like, "We should provide increased police protection in vulnerable neighborhoods" or "We should initiate programs to eliminate the causes of the crimes" (p. 123). The death penalty conclusion does not even seem to be appropriate, since theft and robbery are not capital crimes. Similarly, in the case of the welfare system argument, some other solutions to the problem would seem appropriate to consider, before leaping to the radical solution of eliminating the system altogether. In both cases, the fallacy is marked by a logical leap from the premise to a conclusion that is a long way away from it, rather than considering other solutions that are not so 
far removed from the conclusion.

Is missing the point the same fallacy as wrong conclusion? At first it seems not, because the examples seem different. In the relief of the poor example, as well as in the horrible crime of murder example, the conclusion to be proved is a comparatively specific proposition whereas the actual conclusion is comparatively abstract or general. The pattern is different in the death penalty and welfare system examples. In these cases the conclusion is specific enough, perhaps even more specific than the premise. The problem is that the argument leaps too quickly to it, without even considering more plausible alternatives, and without making much of a case for it as a plausible conclusion to be drawn from the evidence offered. Yet in both cases, it does seem that the fallacy could correctly be described as "wrong conclusion".

\section{The Red Herring Fallacy}

There are many cases where it has been claimed in the logic textbooks, and other sources on critical thinking, that a fallacy of relevance has been committed, even though the argument in the given case does not fit the wrong conclusion model. These are cases where the arguer wanders off the point, trying to divert the audience with a rousing emotional appeal, even though it may not be clear what he is trying to prove. These kinds of cases are classified under the heading of the red herring fallacy. There are many cases in which the red herring fallacy does not consist in an argument to the wrong conclusion, but it merely an attempt at distraction that leads off to a different issue or even on a distracting trail to nowhere. Indeed, Hurley actually provides an example of the red herring fallacy that seems to fit such a description (2003, p. 132).

\section{The Parking Example}

Professor Conway complains of inadequate parking on our campus. But did you know that last year Conway carried on a torrid love affair with a member of the English department? The two used to meet every day for clandestine sex in the copier room. Apparently they didn't realize how much you can see through that fogged glass window. Even the students got an eyeful. Enough said about Conway.

The argument in this case could perhaps be classified as an attempt to attack Conway's character, and so fall into the ad hominem category. But what conveys the strategy of the argument better is that it is meant to excite the interest of the listener by presenting a story that is stimulating, but doesn't really lead to any conclusion about the parking issue. Indeed, it leads away from the inadequate parking issue by interposing the distracting story about clandestine sex in the copier room. If this analysis of the parking example is right, it shows that there are 
cases of the red herring fallacy that are not cases of the fallacy of wrong conclusion.

In the classification system of Walton (2004, p. 243), both the wrong conclusion and red herring fallacies are subsumed under the general category of what is called misdirected argumentation, or argument directed other than along a path of argumentation leading towards the conclusion to be proved. Sometimes the path leads to the wrong conclusion (one other than the one that is supposed to be proved). In such a case, the fallacy of wrong conclusion has been committed. Sometimes the path leads away from the conclusion to be proved, but not to any specific alternative conclusion. The shared structure of these different types of fallacies of irrelevance is indicated in Figure 1 below.

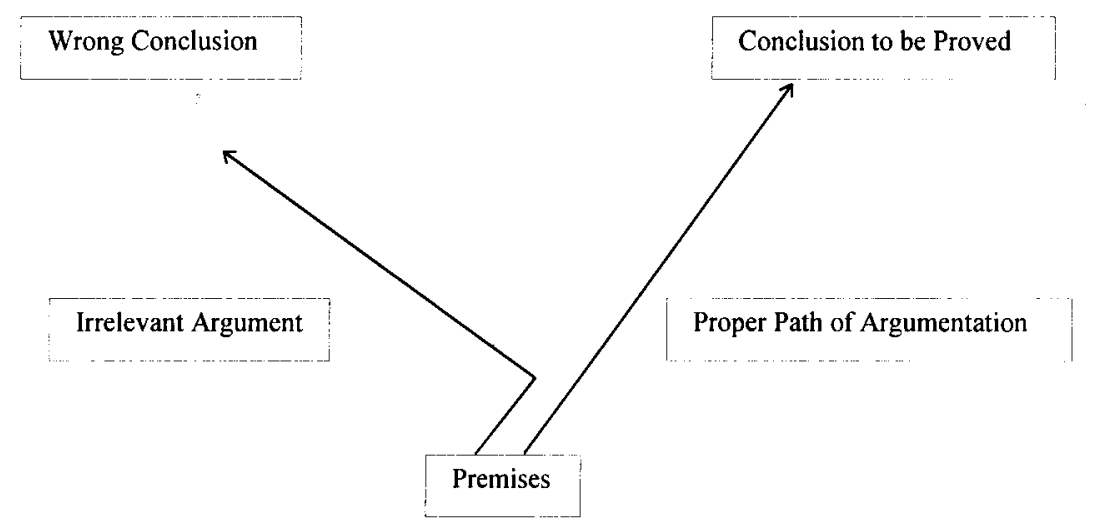

Figure 1: The Structure of Fallacies of Irrelevance

If the line of argumentation has as its end point a specific conclusion that can be identified and that is one other than the conclusion to be proved, the fallacy is that of wrong conclusion. If the line of argumentation initially looks like it is moving toward the designated conclusion to be proved, but then it strays in a different direction, but does not have that conclusion as its end point, the fallacy of relevance committed in the case can be classified as a red herring.

There are two subtypes of red herring fallacy recognized in the analysis of Walton (2004). When it goes to some colorful issue that will get the audience excited and involved, thus distracting them, the fallacy is that of diversionary irrelevance. When the speaker uses a gaseous cloud of rhetoric that doesn't prove some conclusion other than the one to be proved, or doesn't even stick to a single 
well-defined diversionary issue, the fallacy of irrelevance is called pettifogging. The argumentation in the parking example would be classified under diversionary irrelevance, because the argument moves to the allegation of the sexual incident in the copier room. The description of this alleged incident is colorful and distracting. It provides a diversion away from the issue of inadequate parking, distracting the audience. In other cases however, the speaker merely rambles on, perhaps talking about several issues unrelated to the conclusion to be proved, or perhaps even wandering around so much that no single diversionary issue can be identified. These cases fall under the category of pettifogging irrelevance.

\section{Four Examples}

The red herring fallacy is said to be committed when an arguer "diverts the attention" of the listener by "changing the subject to some totally different issue" (Hurley, 1994, p. 127). Hurley's two leading examples in the 1994 edition are quoted below (p. 127).

\section{The Coffee Example}

Your friend Margie says that Tasters Choice coffee tastes better than Folgers. Apparently she is ignoring the fact that Tasters Choice is made by Nestle, and Nestle is the company that manufactured that terrible baby formula for third world countries. Thousands of babies died when the dry milk formula was mixed with contaminated water. Obviously your friend is mistaken.

\section{The Light Bulb Example}

The Consumers Digest reports that GE light bulbs last longer than Sylvania bulbs. ${ }^{7}$ But do you realize that GE is this country's major manufacturer of nuclear weapons? The social cost of GE's irresponsible behavior has been tremendous. Among other things, we are left with thousands of tons of nuclear waste with nowhere to put it. Obviously, the Consumers Digest is wrong.

Just as with Hurley's death penalty and welfare system examples of the missing the point fallacy, and with the relief of the poor and horrible crime of murder examples of the wrong conclusion fallacy, here too there is a leap to a conclusion other than one of a kind that would be justified by the premises given. Thus one might wonder what the difference is supposed to be between the wrong conclusion fallacy and the red herring fallacy. The difference appears to be that in the coffee and light bulb examples, there is a distraction by moving to a controversial issue of a kind that might catch up the listener and attract or divert her attention to this 


\section{Douglas Walton}

issue. In the light bulb example, listeners might be prejudiced against GE, once the company is portrayed negatively as one directed by polluters who irresponsibly produce and mismanage nuclear waste material. Listeners might presumably get excited about the nuclear waste issue and forget that the real issue to be decided in the example is which light bulbs last longer. Similarly, in the coffee example, the baby deaths issue is emotionally compelling, and hence it may easily distract many listeners from the real issue of which coffee tasted better. In both examples, the argument introduces a red herring, a new issue that presumably will be very stimulating to the audience.

In the eighth edition of $A$ Concise Introduction to Logic (2003) the death penalty example and the welfare system example were still being used as the leading examples of the missing the point fallacy. But the coffee example and the light bulb example were replaced by the following two leading examples of the red herring fallacy.

\section{The Electricity Example}

Environmentalists are continually harping about the dangers of nuclear power. Unfortunately, electricity is dangerous no matter where it comes from. Every year hundreds of people are electrocuted by accident. Since most of these accidents are caused by carelessness, they could be avoided if people would just exercise greater caution.

\section{The Pesticides Example}

There is a good deal of talk these days about the need to eliminate pesticides from our fruits and vegetables. But many of these foods are essential to our health. Carrots are an excellent source of vitamin A, broccoli is rich in iron, and oranges and grapefruits have lots of vitamin C.

In these two examples, the fallacy is even more apparent. In the electricity example, it is made clear in the first statement that the argument that follows is supposed to be a refutation of the claim made by the environmentalists that nuclear power is dangerous. However, the rest of the argument is about the dangers of electricity. In the pesticides example, it is made clear in the first sentence that the argument that follows is supposed to be a refutation of the claim that there is a need to eliminate pesticides from our fruits and vegetables. But the rest of the argument is about the nutritional value of fruits and vegetables. In each case, the line of argumentation switches to a different issue, and the argumentation offered is of no use in proving the conclusion stated in the very first sentence. It is fairly clear why each argument can be classified as a red herring. 
What is supposed to be the defining difference between the wrong conclusion fallacy (or missing the point fallacy, as Hurley calls it) and the red herring fallacy? The wrong conclusion fallacy requires the following three characteristics to be present in the argumentation in the given case.

\section{The Three Defining Characteristics of the Wrong Conclusion Fallacy}

1. There is a given set of premises and a specific conclusion that can be identified, that is actually argued for on the basis of these premises. Let's call it the actual conclusion.

2. There is a specific conclusion that is the proposition that is supposed to be argued for in the case. Let's call it the proper conclusion.

3.The actual conclusion is a different one from the proper conclusion.

The presence of each of these three characteristics marks an instance of the wrong conclusion fallacy. Hence in cases of the wrong conclusion fallacy, like the horrible crime of murder example, the tax reform example, the death penalty example and the welfare system examples, there is a hasty leap from the given premises to the wrong conclusion.

The red herring fallacy is also a fallacy of relevance, in that there appears to be a line of argumentation from what is given as the argument to a proper conclusion, and the argument is diverted in a different direction, onto a line of argumentation that may seem to go towards some different conclusion. Because of this overlap of strategy of diversion away from a given line of argumentation, perhaps the red herring fallacy could be seen as a special case of the wrong conclusion fallacy. But there is a difference that might be cited. The wrong conclusion fallacy seems more like a bad shot, a blunder, or error of reasoning. Something is hit, but it is the wrong conclusion. The red herring seems more like a deliberate deception of the listener by the arguer by bringing in some controversial issue that will likely be stimulating and thus distracting to the listener. The red herring seems more like a dialectical fallacy in which one speech partner tries to get the best of the other by using a strategy meant to deceive. It is not just that one has hit the wrong conclusion. Instead, one has taken a whole different direction that leads away from the proper conclusion but leads to some other issue that will excite the listener. In both examples of the red herring fallacy, there is a specific issue, a decision to be made which of the two products is better in some respect. Which bulb lasts longer? Which coffee tastes better? The given argumentation, however, goes off in a different direction by attacking the company that manufactures each product. In each example, the argument offered might be reason for not buying one of the products being considered (or it might not). But it is still not relevant to deciding the specific issue being discussed. 
On the other hand, looking at the relief of the poor example and the horrible crime of murder example, the fallacy seems to be more than just a bad shot or hasty leap to the wrong conclusion. In all these cases, there is some evidence that the speaker is trying to distract the audience by going on to some different issue, perhaps a more abstract or general one, on which the speaker expects the audience will agree. This is an element of diversion. Thus, there is a problem about classifying the examples above when it comes to clearly and definitely putting one group under the wrong conclusion category and the other group under the red herring category.

\section{Analyzing the Four Examples}

To get a more precise analysis of the argumentation in these examples and thereby pinpoint the problem, it is useful to construct argument diagrams for some of them. Let's begin with the pesticides example. The explicit statements that make up the argument are represented in the key list below.

\section{Key List for the Pesticides Example}

(A) There is a need to eliminate pesticides from our fruits and vegetables.

(B) Many fruits and vegetables are essential to our health.

(C) Carrots are an excellent source of vitamin A.

(D) Broccoli is rich in iron.

(E) Oranges and grapefruits have lots of vitamin C.

Evidently the argument is meant to refute $A$. Thus the whole subsequent line of argument that supports $B$ is meant to furnish such a refutation. But it does not. It goes off to a different line of argumentation altogether. The structure of this line of argumentation is displayed in the screen shot of the Araucaria window below. The propositions in the argument are highlighted in the box on the left, and the structure of the argument is displayed on the right. The linked argument with premises $\mathrm{C}, \mathrm{D}$ and $\mathrm{E}$ supports $\mathrm{B}$, while $\mathrm{B}$ is supposedly presented as a refutation of $\mathrm{A}$. 


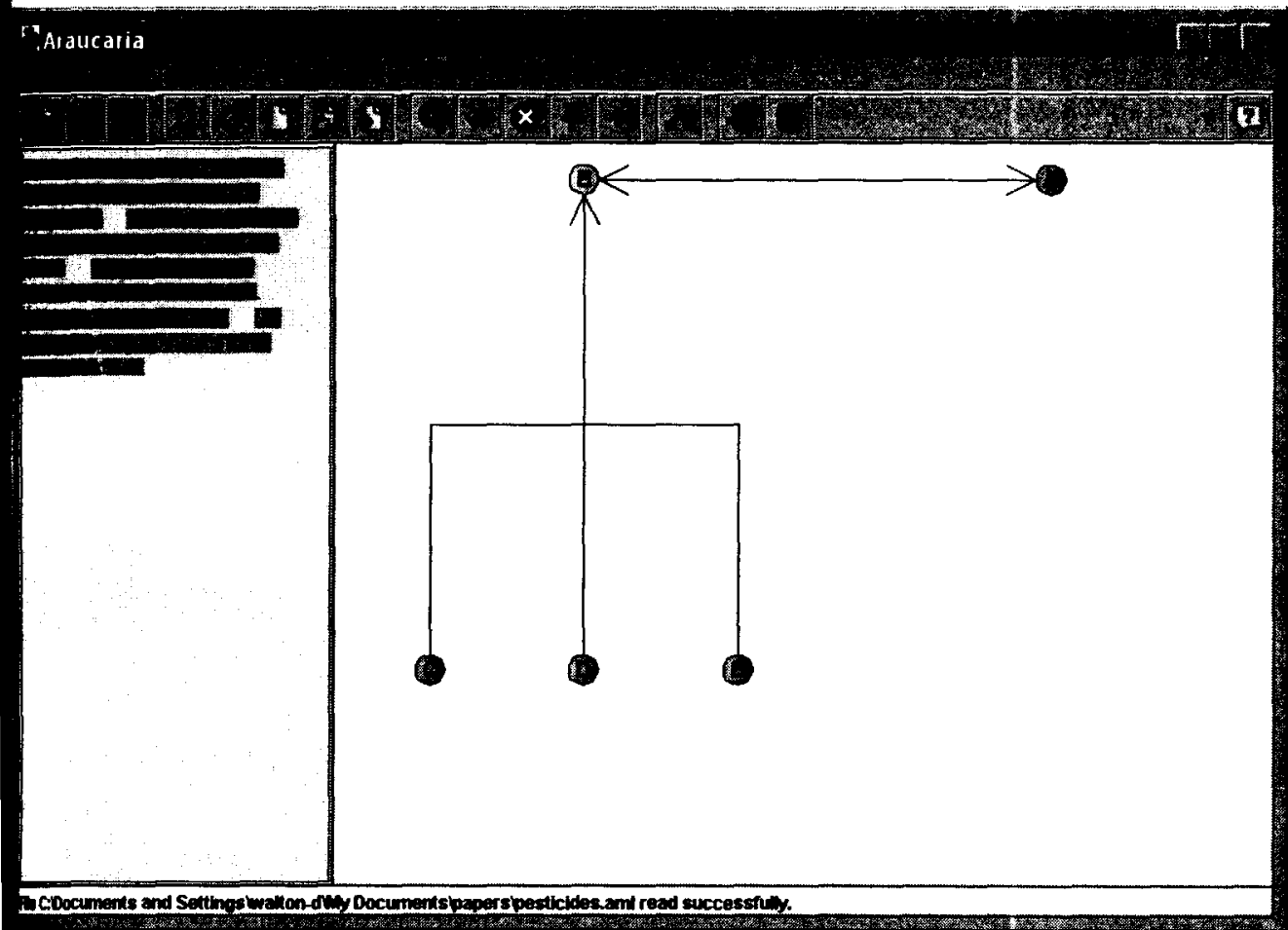

Figure 2: Araucaria Window of the Pesticides Example

This example is an instance of the red herring fallacy because the whole line of argumentation in the example supports $B$ very well. But $B$ does not refute $A$. $B$ is not relevant to $A$. It is the claim that fruits and vegetables are essential to our health. This claim is not only highly plausible, but the remaining statements about the carrots, oranges, and so forth, give reasons to support it. Thus we do have a good argument here, except that what it proves, $(B)$, is not relevant to the conclusion that is supposed to be proved. The fallacy is clearly a failure of relevance. Incidentally, this example is a good one to illustrate the original meaning of the Aristotelian name for the fallacy, ignoratio elenchi, or ignorance of refutation. $B$ fails to refute $A$, and that failure is at the heart of the fallacy.

Much the same kind of analysis can be given by diagramming the electricity example. The coffee and light bulb example also appear similar, as failures of relevance, but in some respects they are different. The precise failure of relevance in these cases is subtler, and more difficult to pinpoint. Even though these examples would prove more challenging for students, they are worth analyzing as fallacies of relevance. Let's choose the coffee example to diagnose the interesting features shared by the two cases. 
To begin to analyze the coffee example beyond the remarks made above, let's try to identify the statement representing the conclusion that is supposed to be proved in the example. Two statements are made that point to this conclusion. One is, "Your friend Margie says that Tasters Choice coffee tastes better than Folgers". The other is, "Obviously your friend is mistaken". The statements, taken together, suggest the argumentation in the example is deigned to refute the statement, "Tasters Choice coffee tastes better than Folgers". In other words, the argument is meant to prove that Margie was wrong when she claimed that Tasters Choice tastes better than Folgers. In this respect, the coffee example looks similar to the pesticides example.

To take a first pass at diagramming the coffee examples, let's identify the premises and conclusions of it in the key list below.

\section{Key List for Analysis 1 of the Coffee Example}

(A) Your friend Margie says that Tasters Choice coffee tastes better than Folgers.

(B) Apparently she is ignoring the fact that Tasters Choice is made by Nestle.

(C) Nestle is the company that manufactured that terrible baby formula for third world countries.

(D) Thousands of babies died when the dry milk formula was mixed with contaminated water.

(E) Obviously your friend is mistaken.

In Figure 3 below, the full text diagram for analysis 1 of the coffee example, $E$ appears on a line horizontal with $A$, indicating that $E$ is a refutation of $A$. 


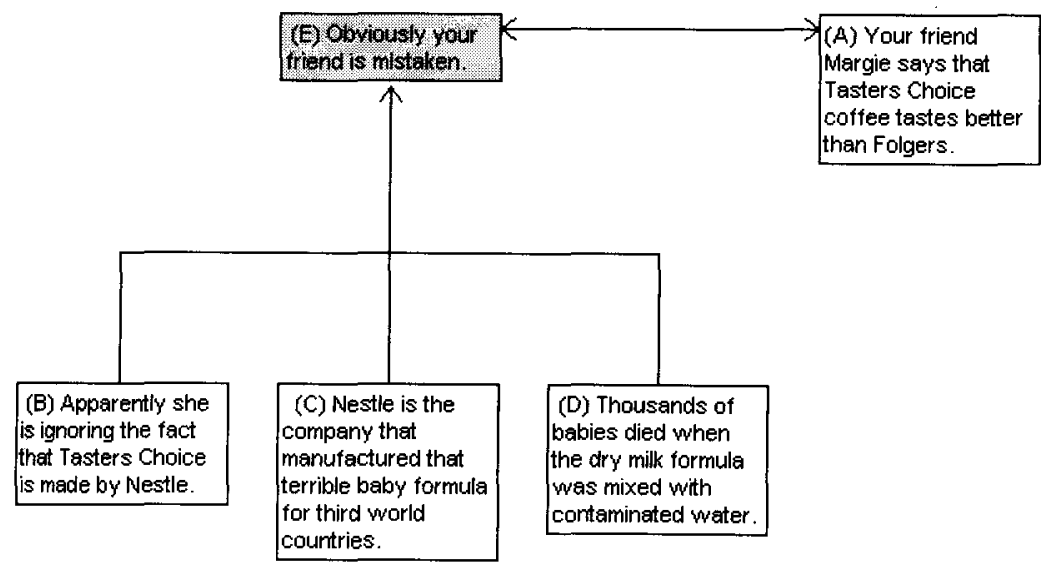

Figure 3: Araucaria Diagram for Analysis 1 of the Coffee Example

The remaining statements in the example form a linked argument supporting $E$. Analysis 1 is a relatively straightforward way of representing the argumentation in the coffee example. Indeed, on this analysis, the argumentation supporting $E$ looks relevant, for it forms a linked argument that supports $E$. Since $E$ is diagrammed as a refutation of $A$, it looks like the argumentation supporting $E$ should be relevant. However $E$ does not really refute $A$. It is only an apparent refutation, so to speak.

There is a deeper way of analyzing the argumentation in the coffee example as an enthymeme that brings out some implicit statements in it. Consider the following analysis of the coffee example. The issue in the coffee example is whether Tasters Choice tastes better than Folgers. But the rationale for bringing in the babies dying story is to offer the listener a reason not to buy any product made by Nestle. The reason given is that Nestle manufactured the "terrible baby formula" that contributed to this tragic outcome. The conclusion of this argument is "You shouldn't buy Tasters Choice". But this whole line of argumentation is irrelevant. The issue is not whether you should buy Tasters Choice but whether Tasters Choice tastes better than Folgers. To diagram this analysis, we begin by identifying three statements explicitly given as premises and conclusions in the text of the example. These are given as $A, B$ and $C$ in the key list below. Then let us try to identify any implicit premise or conclusions that the argumentation in the example could rely 


\section{Douglas Walton}

on to move towards this conclusion or a different one. Following the analysis of the argumentation in the coffee example presented in the previous section, other implicit links in the chain of argumentation have been added.

\section{Key List for Analysis 2 of the Coffee Example}

(A) Tasters Choice is made by Nestle.

(B) Nestle is the company that manufactured that terrible baby formula for third world countries.

(C) Thousands of babies died when the dry milk formula was mixed with contaminated water.

(D) Thousands of babies dying from a terrible baby formula is a bad consequence.

(E) If an action leads to bad consequence, you should not carry out that action.

(F) Buying any product made by Nestle leads to bad consequences.

(G) You should never buy any product made by Nestle.

(H) You shouldn't buy Tasters Choice.

The line of argumentation can be analyzed as follows. $H$ is the ultimate conclusion. The basic reason offered to support $H$ is $G$, combined with $A$. This argument is linked. $G$ is a generalization that fits with $A$ to yield $H$ as conclusion.

(G) You should never buy any product made by Nestle.

(A) Tasters Choice is made by Nestle.

(H) You shouldn't buy Tasters Choice.

This argument is deductively valid, assuming "any" is taken to mean "all without exception". But what should be said about the rest of the argument?

It appears that $F$ and $E$ are offered as reasons to support $G$. But what reasons are given to support $F$ ? It would appear that $B, C$ and $D$ combine to make up a linked argument that supports $F$. The following Araucaria diagram displays the argumentation structure. 


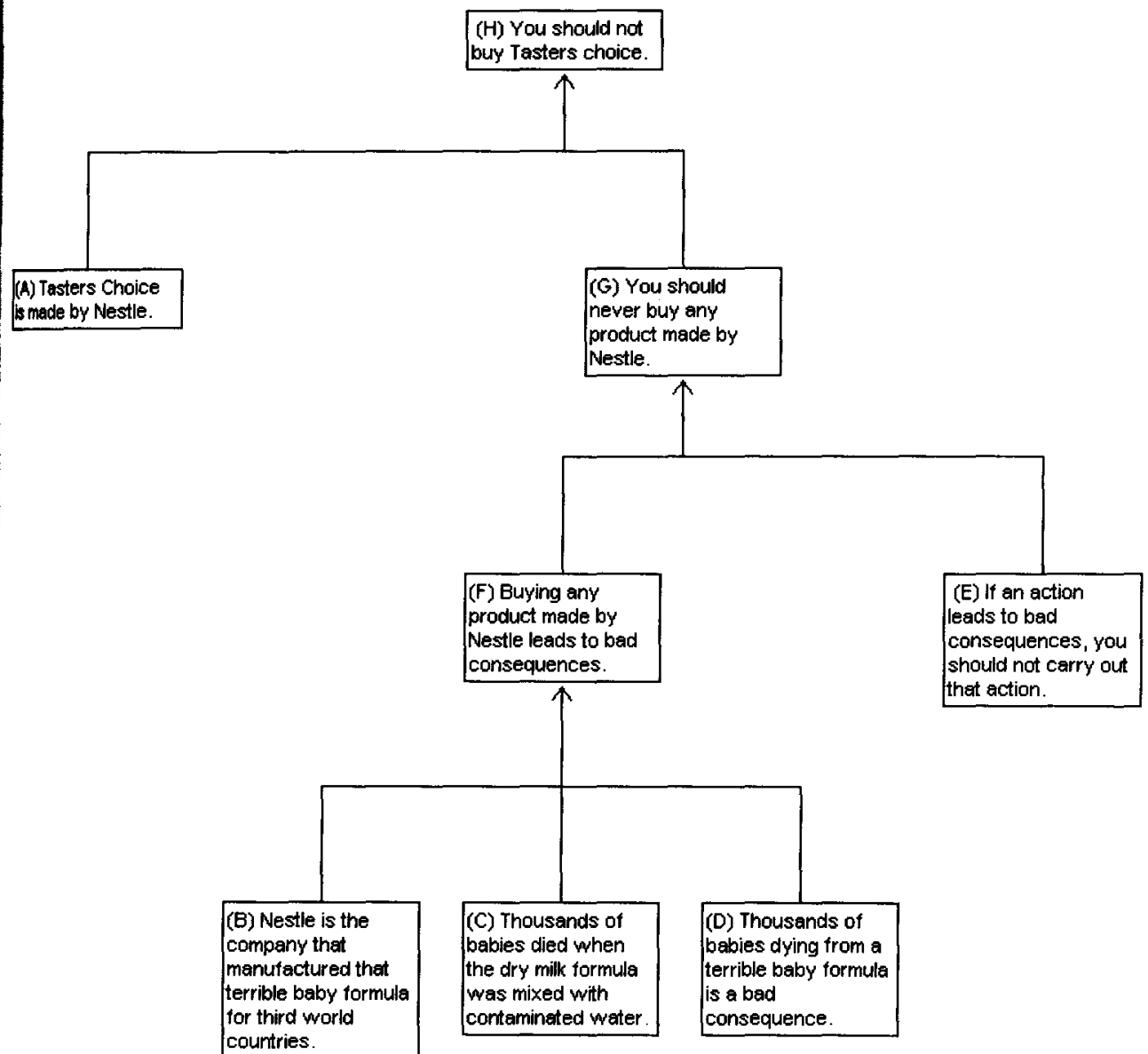

Figure 4: Araucaria Diagram for Analysis 2 of the Coffee Example

The diagram in Figure 4 represents the red herring fallacy in a different way from the diagram in Figure 3, but in both instances the failure can be seen as one of a line of argumentation that fails to support or refute the conclusion at issue. The argumentation represented in Figure 4 is based on an analysis that makes several 
implicit assumptions explicit, namely $E, F, G$ and $H$. However, Figure 4 shows an argument that (at least arguably) does offer good reasons to support the conclusion $H$. But since there is no line of argumentation from $H$ that gives good reasons to support the conclusion that one coffee tastes any better or worse than the other, the whole network of argumentation represented in Figure 4 is irrelevant.

Up to this point, it has been shown that these examples are genuinely interesting cases of kinds of failures of relevance associated with the fallacies of missing the point (wrong conclusion) and red herring. Although both are fallacies of relevance, there does seem to be a difference between them of a kind that students could identify. But still, the two fallacies are not all that different from each other. Could there be some more precise criterion for distinguishing between them? To study this question, it is necessary to take a closer look at the examples and the basis for their differentiation.

\section{Classifying the Examples}

There are various problems with classifying the fallacies of relevance in these examples. The first one is that it can plausibly be argued that the fallacy in the coffee example is basically the same fallacy that is committed in both the death penalty and welfare system examples and classified as the fallacy of wrong conclusion (missing the point). As represented by the diagram in Figure 4, the conclusion of the argument is the statement that you shouldn't buy Tasters Choice. But that isn't the real conclusion that is supposed to be proved by the argument. The real conclusion that should be proved by any relevant argument given is that Folgers tastes better than Tasters Choice. In other words, the fallacy committed in the coffee example, according to analysis 2 , is that the premises of the given argument support one conclusion, but then a different conclusion, vaguely related to the correct conclusion, is drawn. But that analysis corresponds exactly to Hurley's definition of the fallacy of missing the point, or the fallacy of wrong conclusion as it might well be called. It looks like a comparable analysis of the other three examples of red herring can be given. Consider the light bulb example. The conclusion that is supposed to be proved is whether Consumers Digest is right or wrong on what it claims about the one brand of light bulb lasting longer than the other. But what argument is actually given? The argument cites thousands of tons of nuclear waste supposedly produced by GE's "irresponsible behavior". So what does this argument prove? What conclusion does it support, or lead to? Well, it might plausibly enough support the conclusion that it would be a bad thing to buy GE products, since such an action would be linked to the bad outcome of nuclear waste by adding to GE's profits. But once again, it can be argued, this is the wrong conclusion. The conclusion that needs to be proved is the proposition that GE's light bulbs don't last as long as their competitor's. In short, it looks like a very good case can be made for classifying the argument in the light bulb example as committing the fallacy of wrong conclusion. If this is the case, there is a general 
problem with all these cases. The arguments that were supposed to fit the model of the red herring fallacy actually fit the model of the wrong conclusion (missing the point) fallacy.

Another problem is that the later examples, the electricity and the pesticides ones, are different from the earlier ones, the coffee and the light bulb examples, in a certain respect. There is a factor present in the latter examples that does not seem to be present in the former ones. This factor is made especially evident in analysis 2 of the coffee example, but it is also apparent in the light bulb example. One might observe that in both cases there is a shift from a discussion about the truth or falsity of a proposition to a different kind of dialogue about action. This secondary type of dialogue could be classified as a deliberation about what course of action to take. In the coffee example, the conclusion that is supposed to be argued for is the proposition that Margie's statement "Tasters Choice coffee tastes better than Folgers" is mistaken. The claim made is that a certain specific statement is false. However, on the analysis outlined above, the argumentation offered is to the effect that it would be a bad idea to buy Folgers coffee because Nestle is the company that made that terrible baby formula that was responsible for thousands of babies dying. This line of argumentation is against a particular course of action, namely that of buying Folgers coffee. It is about choosing which type of coffee to buy, a decision about actions. Thus one could say there has been a dialectical shift in the argumentation from a critical discussion type of dialogue about whether a particular statement is true or false, to a deliberation type dialogue about which action among a set of alternatives is right. This factor is not present, at least so markedly, in the electricity example and the pesticides example. There, the issue is about actions, to some extent. At any rate, there is not such a comparable sharp shift from a critical discussion to a deliberation type of dialogue in the two later examples.

The two newer examples of red herring do seem to be different from the two older ones. It seems like the two newer ones are easier to classify as instances of red herring as opposed to falling into the wrong conclusion category. The hardest problem, as shown very pointedly in analysis 2 of the coffee example, is to classify the coffee and the light bulb examples as instances of the red herring fallacy that do not collapse into being instances of wrong conclusion of the kind exemplified by the death penalty example, the welfare system example, the relief of the poor example, and the horrible crime of murder example as well. This problem also applies to the electricity and the pesticides examples. The conclusion to be proved in the electricity example is that nuclear power is dangerous, whereas the conclusion argued for is that electricity is dangerous. The conclusion to be refuted in the pesticides example is that there is a need to eliminate pesticides from our fruits and vegetables, whereas the conclusion argued for is that these foods are essential to our health.

What classification system is best, given the analyses of these examples 
presented above? Can any classification system cope with the problems exposed by the various analyses of these examples? Can it be argued that all the examples commit the wrong conclusion fallacy? If so, should that hypothesis, in turn, lead to the conclusion that the red herring fallacy is best classified as a subspecies of the wrong conclusion fallacy? Or is the best system of classification one that holds wrong conclusion and red herring to be two distinct categories of fallacy of relevance? Copi and Cohen $(2004$, p. 51$)$ classified the red herring fallacy as a subspecies of the irrelevant conclusion (wrong conclusion) fallacy. At least, they include it under the general heading of the fallacy of irrelevant conclusion or mistaken proof, said to be committed when an argument "purporting to establish a particular conclusion is instead directed to proving a different conclusion" (p. 50). Hurley treated missing the point (ignoratio elenchi) in a different section from red herring, implying they are two different fallacies. However, he wrote (2003, p. 124) that the red herring fallacy is "closely associated" with the fallacy of missing the point. A general problem is which system of classification is best.

Another problem of classification is that according to analysis 2 of the coffee and light bulb examples, these two cases come out as instances of the wrong conclusion fallacy, even though they do have some elements of red herring as well. One solution is to bite the bullet and conclude that, initial appearances to the contrary, these two examples should really be classified as instances of the wrong conclusion fallacy, at least on analysis 2 , which portrays them as using argumentation from negative consequences. This solution could possibly be defended, as long as a firm distinction can be maintained between analyses 1 and 2 of both cases. On analysis 1 , the fallacy committed is that of red herring. But on analysis 2 , a case can be made for classifying both as instances of wrong conclusion. Another problem is that in the relief of the poor and the horrible crime of murder examples, there is some evidence of a red herring strategy. The speaker diverts the argumentation toward discussion of a more abstract issue on which he might expect audience agreement, or which he might expect to provoke audience interest. Still, one might argue, because there is a definite wrong conclusion that the arguer supposedly proves, or least persuasively argues for, the central fallacy is that of wrong conclusion.

To address these classification problems, let's begin by reviewing the three defining characteristics of the wrong conclusion fallacy, and then review what we found to be the leading characteristics of cases of the red herring fallacy.

\section{The Three Defining Characteristics of the Wrong Conclusion Fallacy}

1. There is a given set of premises and a specific conclusion that can be identified, that is actually argued for on the basis of these premises. Let's call it the actual conclusion.

2. There is a specific conclusion that is the proposition that is supposed 
to be argued for in the case. Let's call it the proper conclusion.

3.The actual conclusion is a different one from the proper conclusion.

The presence of all three characteristics in a case marks an instance of the wrong conclusion fallacy in that case. We took the examples of the missing the point fallacy to share these three characteristics. In Hurley's examples of the missing the point fallacy, like the death penalty example and the welfare system examples, there was a characteristic hasty leap from the given premises to the wrong conclusion. Hence we classified these cases as conforming to the three defining characteristics of the wrong conclusion fallacy. We classified the missing the point fallacy as special subcategory of the wrong conclusion fallacy.

The main problem with the examples of the red herring fallacy we examined was that they (arguably) fit these three characteristics as well. In the electricity example, the proper conclusion is that nuclear power is not dangerous. The actual conclusion is that electricity is dangerous. In the pesticides example, the proper conclusion is that pesticides do not need to be eliminated from our fruits and vegetables. The actual conclusion is that fruits and vegetables are essential to our health. The coffee and the light bulb examples also arguably exhibit all three characteristics of the wrong conclusion fallacy, especially on the analysis 2 type of reconstruction of the argumentation in the case.

\section{Two Hypotheses: Strategic Diversion and Probative Weight}

However, even conceding these common elements, we did notice a key difference between the examples classified as wrong conclusion and as red herring. In the examples of the wrong conclusion fallacy, it seemed like the fallacy committed was more like a bad shot, a blunder, or error of reasoning. In the examples classified as red herring, the fallacy seemed more like a deliberate deception of the audience by the arguer's bringing in some controversial issue that is stimulating and distracting to the audience. On the basis of this way of dividing the two fallacies, the red herring seems more like a dialectical fallacy in which the arguer uses a strategy of diversion meant to deceive the audience. Instead of merely hitting the wrong conclusion inadvertently, the arguer has deliberately gone off in a different direction to another issue that is meant to excite the audience and thereby lead it away from worrying about proving the proper conclusion. In the coffee example and the light bulb example of the red herring fallacy, there is a decision to be made about which of the two products is better in some respect. Which bulb lasts longer? Which coffee tastes better? But the arguer provides an exciting interlude by attacking the company that manufactures each product on ethical grounds. Similarly, in the electricity and pesticides examples, the use of the dramatic interlude distracts attention away from the issue set for the discussion. Even though it has been argued on analysis 2 that the fallacy committed in the 


\section{Douglas Walton}

coffee and light bulb examples is that of wrong conclusion, there is an additional dimension of strategic diversion in these cases. The additional dimension is the strategy of leading the listener off into a different controversy, which he or she is likely to find very controversial and diverting. In the coffee and light bulb examples, the argument to the wrong conclusion could be especially attractive as a distraction in that it offers what would be taken to be good reasons for not buying the product in question for politically activist listeners who are eager to support a policy of boycotting commercial products for "ethical" reasons.

Along these lines it could be argued that it is this strategic device of diversion characterizing the red herring fallacy in the coffee example, the light bulb example, the electricity example and the pesticides example that sets them off from the relief of the poor example and the horrible crime of murder example. Yes, in the former cases, the three identifying characteristics of wrong conclusion are present, but what sets them off from the latter cases is the element of diversion by the strategy of introducing an interlude of argumentation that leads off in a direction away from the proper path of argumentation to the proper conclusion.

Another hypothesis can be used to analyze the difference between the wrong conclusion cases and the red herring cases. According to this hypothesis, the difference is that in the wrong conclusion type of case, something is proved, whereas in the red herring type of case, the interlude may provide a mass of argumentation of some sort, but this mass is not used to prove anything. The key characteristic of the red herring cases is that these arguments do not really go anywhere, as far as proving a specific conclusion is concerned. They provide an interlude of argumentation. But the chain of argumentation doesn't prove anything, at least anything significant in relation to the conclusion to be proved. It doesn't prove that proposition, nor does it prove one that is different it but looks similar. The interlude argumentation is dead weight. ${ }^{8}$

This analysis depends on what is meant by the expression "proving something", in a sense that requires something more than just a valid (or structurally correct) argument. This idea can be expressed in more precise terms by introducing the notion of probative weight-a concept of argument evaluation. The idea is that if premises have probative weight, and the argument from these premises to a conclusion is structurally correct, then the premises can throw probative weight onto the conclusion. An argument can be structurally correct if it is deductively valid, inductively strong, or if it fits the structure of a presumptive argumentation scheme. In such a case, the probative weight of the conclusion is increased as a function of two factors of the argument: (1) the probative weight of the premises, and (2) the probative weight (structural strength) of the argument from the premises to the conclusion. This type of case represents an increase in probative weight of a conclusion due to an argument supporting that conclusion. There is also the kind of case where an argument decreases the probative weight of a conclusion by refuting (or undercutting) the conclusion. Either type of argument is probatively 
relevant. Probative relevance is contrasted with topical relevance in (Walton, 2004). For example, the proposition 'All bananas are yellow' is topically relevant to 'The yellow hat is in the window', but is not probatively relevant to it. On the other hand, 'All bananas are yellow' is probatively relevant to 'This banana is yellow', because the premise can throw probative weight onto the conclusion. A probatively relevant argument can increase or decrease the probative weight of its conclusion, whereas an argument that is topically relevant but not probatively relevant does not have this capability.

Probative weight can be represented on an argument diagram in Araucaria by assigning values to the nodes and the arrows in the diagram (Reed and Rowe, 2003 , p. 15). You can assign probability values between 0 and 1 , or you can annotate nodes and arrows in an argument with markers of plus, minus or zero, in a many-valued logic. Assigning evaluations of this sort marks the initial probative weights attributed to each of the components of an argument. Changing the values of some parts will require changes in other parts. Various rules can be used to make such adjustments of probative weight in a diagram. One is the least plausible premise rule which states that the conclusion of a deductively valid argument must have at least as much plausibility as the least plausible premise. Take the example of an argument that has the form modus ponens, and one premise has a value of .8 , the other a value of .6. Let's say that the conclusion has a relatively low initial value of .3. Using the least plausible premise rule, the new value that must be assigned to the conclusion is that of .6. The least plausible premise rule can also be applied to arguments that are not deductively valid, but where the argument is inductive or presumptive, the conclusion following from the premises by a presumptive argumentation scheme. This can be done by assigning a value to the conditional that functions as the warrant of the argument. This value can then be factored in, when applying the least plausible premise rule. In a convergent argument, the conclusion needs to be revised upward to the value of the most plausible premise. In a linked argument, the probative weight of the conclusion needs to be revised upward to that of the least plausible premise.

Given this notion of probative weight, a distinction can be drawn between how the argumentation works in the wrong conclusion examples versus the examples of red herring. In the horrible crime of murder example, the argumentation presented by the prosecution does (we may presume) throw probative weight onto the conclusion 'Murder is a horrible crime'. The problem is that it doesn't throw probative weight on the conclusion 'The defendant is guilty of murder', even though these two propositions are topically relevant to each other. Even worse, once the proposition 'Murder is a horrible crime' is proved, it is different from the proposition 'The defendant is guilty of murder', meaning that there is no evidential route (as would be displayed on an Araucaria diagram) going from the first one to the second, such that the argument increases or decreases the probative weight of the second one. What is meant by saying that the two propositions are "different", 
in the sense of the word used in the three identifying characteristics of the fallacy of wrong conclusion, is that the two are so far apart that there is no chain of argumentation going from one to the other, such that increasing (or decreasing) the probative weight assigned to one also increases (or decreases) the probative weight assigned to the other. Now contrast how probative weight works with cases of wrong conclusion versus cases of red herring.

In cases of red herring, there is an interlude of argumentation that doesn't go anywhere. In the parking example, to cite the first case, there is the whole story of Conway's torrid love affair carried on in the copier room. The problem with this interval is that it doesn't really lead anywhere. Thus it fails to either increase or decrease the probative weight initially assigned to the conclusion, the proposition that Conway's argument that the parking is inadequate on campus is wrong. It fails to refute Conway's argument or to support it. It's not that it goes to another conclusion that is different from the one to be proved or disproved. Its capability to throw probative weight onto the conclusion to be proved, or any proposition that may be different from it but looks similar to it, or close to it, is null. The interlude about the copier room is just dead weight. Of course, you could argue that it suggests that Conway is a bad person, and thus that the argument carries probative weight as an ad hominem attack on Conway's credibility. If so, it is relevant, if it meets the requirement for an ad hominem argument that should carry probative weight. But assuming it is not really an ad hominem argument, or one carrying any probative weight, but merely a digression, then the fallacy in it is rightly classified as a red herring as opposed to a wrong conclusion.

The point can be illustrated even more precisely using the pesticides example. Look back to figure 2, the Araucaria window diagramming the argumentation in the pesticides example. Propositions $C, D$ and $E$ all look highly plausible. They are not really in dispute. Let's assign them high values of $.9, .8$ and .9 respectively. Let's say that the proposition $B$ also has a fairly high initial value. Let's give it a value of .7. And finally, let's say that the probative strength of the linked argument from $C, D$, and $E$ can be assigned a weight of .8. These values are marked on the argument diagram in figure 5 (see next page). Given these values of the premises, and the strength of the linked argument from them to conclusion $B$, using the least plausible premise rule, let's upgrade the value of $B$ to .8 . These values reflect the probative worth of the linked argument from $C, D$, and $E$ to $B$. The problem concerning a potential fallacy of relevance comes in when we examine the relationship of this subargument on the diagram to proposition $A$, the ultimate conclusion that is supposed to be refuted. The problem is that the whole linked argument from $C, D$ and $E$ to $B$ is probatively useless. It doesn't really go anywhere. It is supposed to refute $A$, as the diagram in figure 2 shows, but in fact it does not. It does not affect whatever probative weight we might initially assign to $A$ at all. The proposition that we need to eliminate pesticides from our fruits and vegetables is quite plausible. It, we may presume, has a high degree of probative weight initially. The problem is that the argument to its left in figure 5 does not affect this 


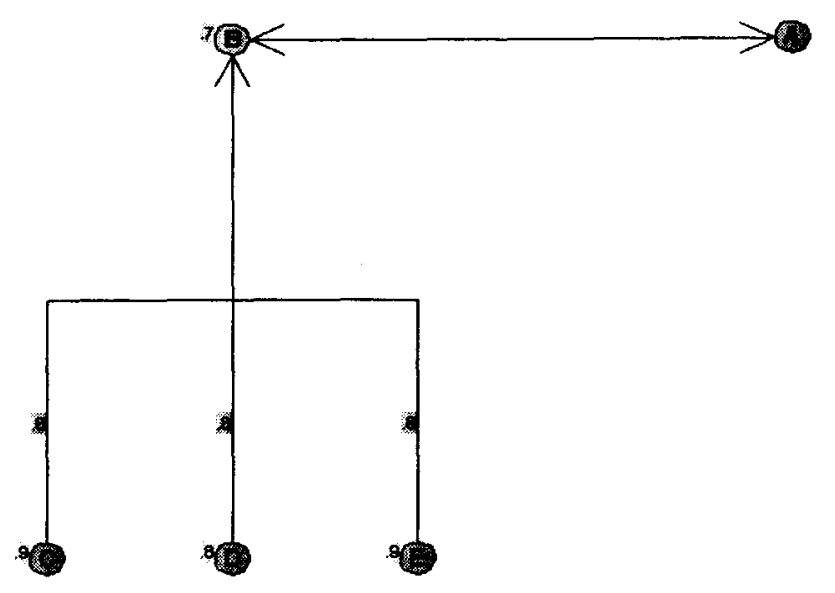

Figure 5: Araucaria Diagram of the Pesticides Example with Probative Weights

initial assignment of value one way or the other. It does not make its probative weight heavier or lighter. Thus it is irrelevant.

In the other examples of red herring, as in the pesticides example, the same kind of analysis using probative weight can be given of the argumentation in the case, showing it to be an instance of the red herring fallacy. The difference between the wrong conclusion fallacy and the red herring fallacy, on this hypothesis, can be diagnosed as follows. In a case of the wrong conclusion fallacy, the given argument has the capability of throwing probative weight onto some conclusion. But it is the wrong conclusion, i.e., it is a conclusion that is different from the conclusion to be proved, even though it may appear similar to it, or may not be distinguished from it by an uncritical audience. However, in a case of the red herring fallacy, the failure is different. The problem here is that the interval, while it may be a colorful and emotionally exciting digression to a "hot" issue, doesn't really go anywhere at all, from a probative point of view. On the diagram, it is a mass of argumentation that may hang together internally, but it fails to connect up with the conclusion that is supposed to be proven in the given case. It fails to either increase or decrease the probative weight initially assigned to that conclusion. Thus what is important for diagnosing the fallacy of relevance in red herring cases is not the exact probative weights that are initially assigned to each of the propositions that figure as premises and conclusions in the argumentation. It is how these initial probative weights are revised upwards or downwards once the whole network of argumentation is connected up, as displayed on an argument diagram. 
On this hypothesis, the method used to diagnose red herring is different from that used to diagnose wrong conclusion. Both involve identifying the premises and conclusion of a given argument. Both involve probative weight. But the modus operandi of each fallacy is different from that of the other. To analyze and evaluate an instance of wrong conclusion, you need to identify the conclusion to be proved in the case, and then identify the conclusion that is actually proved. Then you need to show that the former is different from the latter. To show this, you need to show there is no path of argumentation from the former to the latter. You need to show that the former proposition, even if proved and shown to have strong probative weight itself, does not increase or decrease the probative weight of the proposition that is supposed to be proved. To analyze and evaluate an instance of red herring, the methodology required is different, as indicated above. In this kind of case, you need to identify a digression, or interval containing a sequence of argumentation or indeed any kind of discourse that is simply dead weight when it comes to increasing or decreasing the probative weight of the conclusion to be proved in the case. It may prove a conclusion, as in the pesticides case, but the whole chain of argumentation leading into this conclusion, along with the conclusion itself as part of the interval, is useless to prove the ultimate conclusion that is supposed to be proved in the case. Now we are brought back to the problem of deciding which classification is better.

\section{Formulating Criteria for a Classification System}

One problem for the strategic diversion hypothesis is that there does seem to be some element of strategic diversion present in the examples of wrong conclusion presented in section 2 . At least, such an element is potentially there, depending on how the argumentation in the example is interpreted. In the horrible crime of murder example, it appears likely that the prosecutor is using a rhetorical ploy of going on at length describing in detail how murder is such a gruesome and horrible crime. Similarly, in the other examples, it could well be that the premise of the argument is part of an emotive attempt to arouse the audience on a "hot button" issue like crime, helping the poor, or welfare benefits. Thus one could plausibly argue that these examples all appear to involve the use of strategic diversion which would make them fall into the red herring category rather than the wrong conclusion category. Or at least it makes it difficult to defend a clear binary classification system that would make all these examples fall into the category of wrong conclusion while the remaining examples, like the coffee and electricity examples and so forth, fall into the red herring category. Thus the problem poses an objection to the strategic diversion hypothesis. This hypothesis, according to the objection, doesn't work very well in offering a clear method of classifying the examples. They all tend to come out as instances of the red herring fallacy, leaving nothing, or very little, in the wrong conclusion category.

In reply, it needs to be observed that this objection is based on a certain way of 
interpreting the examples in section 2 . The examples, as presented, are very short. The reader is left to fill in context, or not. Thus in reply to the objection it could be said that, of course, if you fill in enough details about how the arguer went on length talking about the horrible crime of murder, or some other emotively diverting subject, then certainly the example becomes a case of red herring. By such a means of extending the example, you could always make a case of wrong conclusion into one of red herring. But is this extension of the example legitimate? One could argue that it is not, because in judging the argumentation in any example, or real case, we have to go by the text actually presented in the case. It is no good changing the case by filling in what one might take to be background contextual material that might not represent the real argumentation presented in the case. Thus one could say that there is a bit of the straw man in the objection to the strategic diversion hypothesis presented above, because it is reading in a strategic diversion to these examples that might not be there. If it's not there, and there is a wrong conclusion that can be identified in the example, then that example should be classified as wrong conclusion rather than red herring. That is the reply to the objection.

A solution to the problem can be proposed by combining the two hypotheses, forming the following method of drawing the classification between the two fallacies of wrong conclusion and red herring, and applying it to examples. In order for the example to be an instance of wrong conclusion, three criteria have to be met. A "wrong conclusion" is defined as a proposition is different from the conclusion to be proved, but which may look similar enough to it to the audience so that it can be confused with it. The method is based on the following four criteria:

\section{The Four Criteria for Identifying Cases of the Wrong Conclusion Fal-} lacy

(1) There has to be a proposition identified as the wrong conclusion.

(2) The given argument has to throw probative weight onto that wrong conclusion.

(3) The given argumentation leading to the wrong conclusion has to throw no probative weight onto the conclusion to be proved.

(4) There has to be a lack of sufficient evidence of a strategic diversion.

Some comments on how much evidence is needed when applying these criteria will prove useful. The first comment concerns how much probative weight needs to be thrown onto the conclusion by the argumentation in the case. It need only be a little. But it would be arbitrary to try to specify any numerical measure. The second comment concerns how much evidence of strategic diversion is needed. Once again it is not helpful to try to quantify a degree or amount of such evidence required. All one can say is that there has to be definite textual evidence of the kind 
indicated in the examples classified above as red herring. Even if it is possible that the argumentation in the case could be part of a strategic diversion tactic, it is not justified to classify the fallacy as in the case as one of red herring unless definite textual evidence of the diversion given.

The following criteria can now be given for identifying cases where the red herring fallacy has been committed:

\section{The Three Criteria for Identifying Cases of the Red Herring Fallacy}

(1) There has to be no proposition that can be specified as the wrong conclusion.

(2) There has to be sufficient evidence of the existence of a strategic diversion.

(3) The interlude in the strategic diversion has to throw no probative weight on the conclusion to be proved.

Criterion (3) is exhibited as a characteristic of the argumentation shown in figure 5 of the diagram of the pesticides example above. The linked argument on the left throws probative weight onto proposition $B$, but $B$ throws no probative weight at all onto $A$. The whole mass of argumentation leading into $B$ is dead weight, probatively speaking. In this instance, $B$ is supposed to be a refutation of $A$. That is, $B$ is supposed to provide a reason not to accept $A$, or to reject $A$. But it does not. Thus the argumentation in the pesticides case can be classified as an instance of the red herring fallacy.

Although these criteria are designed to draw a clear classification between wrong conclusion and red herring, all four of Hurley's examples of red herring are borderline cases. Although the electricity example is classified as red herring by the probative weight and strategic diversion evidence, the fact remains that one can very plausibly cite evidence from the text of the example that the statement "Electricity is dangerous no matter where it comes from" is a wrong conclusion that is argued for by the rest of the argument. Similarly, in the pesticides example, the evidence from the text indicates that the statement "But many of these foods are essential to our health", is a wrong conclusion supported by the rest of the argumentation that follows it. Thus when you apply to apply the sets of criteria above to these cases, it seems there is good evidence supporting the argument that they should be classified as instances of both the red herring and wrong conclusion fallacies.

The most difficult borderline cases to apply these two sets of criteria to are the coffee and light bulb examples. There certainly is quite strong evidence of the existence of a strategic diversion in both these cases. On analysis 1 , they seem to fit all three criteria for the red herring fallacy very well. But the problem is that, on analysis 2 , there is a proposition that qualifies as the wrong conclusion, and the 
given argumentation can be seen as leading to that conclusion instead of the one to be proved. Consider the analysis of the coffee example represented in Figure 4 for example. The network of argumentation shown in Figure 4 all goes into supporting the conclusion "You should not buy Tasters Choice". If so, there is evidence, at least on analysis 2 , that the coffee example should be classified under the heading of the wrong conclusion fallacy. On the other hand, the network of argumentation represented in Figure 4 can quite justifiably be classified as a diversion. If so, there is some evidence that the coffee example can be classified as an instance of the red herring fallacy, according to the three criteria for that fallacy. So here we have a problematic borderline case. The coffee example, and the same could be said of the light bulb example, are problematic, suggesting that at least in some cases, the same argument could fall into both the wrong conclusion and red herring categories.

The criteria for each fallacy formulated above rule out this possibility, however. There is evidence of a strategic diversion, and so according to criterion (4) of the wrong conclusion fallacy, the coffee example cannot fit this classification. But there is a proposition that can be specified as the wrong conclusion. So according to criterion (1) of the red herring fallacy, the coffee example cannot fit this classification either. According to the criteria formulated above then, the coffee example, on analysis 2 , is neither a case of wrong conclusion nor one of red herring. This seems to leave it in limbo. One possible solution is that these two examples could be classified under a wider category of misdirected argumentation under which both wrong conclusion and red herring fit as subspecies. If this solution is felt to be not specific enough to deal with such cases, another possible solution is to allow for an additional category of diversionary arguments that go to a wrong conclusion. Such a case would have some of elements of red herring and some elements of wrong conclusion.

The problem is certainly a severe one for any attempt to devise a useful classification system, much more severe than seems to have been anticipated by logic textbooks designed to offer readers some clear system of distinguishing between one fallacy and another in common instances. It is perhaps less severe for Copi and Cohen's approach, which takes red herring to be a subspecies of wrong conclusion. It is more severe for Hurley's approach, which takes them to be two separate fallacies. Yet, from the analyses given above, it would seem that they are two distinct and separate fallacies, even though both are pure fallacies of relevance. The wrong conclusion is the easier of the two to pin down. It seems more specific. Once an argument has gone to a wrong conclusion, instead of the right one, the argument has made a definite mistake that can be clearly diagnosed by a critic who says, "Your argument has gone to the wrong conclusion". The red herring fallacy, in contrast, depends on the element of strategic diversion being present in a case, and this is a little hard to definitively determine from the evidence of a case, as shown by our analysis of cases above. Some would say that whether strategic diversion exists in a case depends on the arguer's intentions. However it could be 
countered that what is required is evidence of the arguer's commitments, as judged by the text of discourse of the case. At any rate, wrong conclusion is more open and shut, because it depends only on identifying two propositions in a case, and judging whether they are different or not. Red herring depends on how strong the evidence is in a case showing the presence of a strategic diversion.

A key difference between the two fallacies become apparent when one considers the objections that need to be raised in examples where each one has supposedly been committed. In a case of wrong conclusion, the critic can respond with an affirmative criticism, "You have gone to the wrong conclusion!", whereas in the case of a red herring, it seems more appropriate to ask the question, "Where are you going with this line of argument?" This difference stems from the normal expectation in such cases. You expect the speaker to arrive at some conclusion. If he doesn't, you politely wait to see where she is going, hoping that she will eventually get to the point. With the wrong conclusion fallacy, you know she has got to that point, and that it is the wrong one. With the red herring fallacy, the issue still seems to be open. You do not yet know where she is going, but she seems to be off the point or moving away from it. Thus responding appropriately to the wrong conclusion fallacy seems to allow for or require a refutation, while responding to the red herring fallacy seems to call for a question asking the speaker where she is going with this line of argument. The latter seems more like a procedural move or challenge that could be made, for example, by the chairperson of a meeting.

Because the two fallacies seem different in these fundamental ways, classifying the coffee and the light bulb example as instances of both fallacies does not seem quite right. What is needed is a system that can enable a user to cleanly put examples into one category or the other, either wrong conclusion or red herring. Telling users that many common cases might have to go into both categories seems to be unhelpful, and even potentially confusing. It might be better if we could give the users some other way of classifying cases like the coffee example and the light bulb example, a way that would do justice to the real differences between the two fallacies of relevance.

Finally, it is useful to add a cautionary note about the analyses of these cases above, and what implications can be drawn from them about classification. How the coffee example should be classified remains somewhat subject to interpretation however. In all four of Hurley's problematic examples of red herring, there was evidence of a wrong conclusion that could be found in the analysis of the text of discourse in the example. But this evidence was indirect, and dependent on the analysis. If you examine Figure 4 displaying analysis 2 of the coffee example, you can see that the wrong conclusion, 'You should not buy Tasters Choice' was not explicitly stated in the text of the case. The analysis of the coffee example takes it to be an enthymeme. Four of the propositions in the key list for the Araucaria diagram of the coffee example were implicit premises or conclusions not explicitly stated in the text of the example, as noted in the discussion of that case above 
Because the argument is an enthymeme, on analysis 2 , its classification as an instance of red herring is very much dependent on how the argumentation in the example is analyzed. Thus its classification as an instance of wrong conclusion is conditional on what one takes to be the unexpressed premises and conclusions in the argument. This cautionary note needs to be kept in mind when putting forward a classification system designed to help a user distinguish between wrong conclusion and red herring.

\section{Proposal for a Classification System}

One solution considered in the previous section was to have a category of diversionary arguments that go to a wrong conclusion. A system of classification that would allow for this possibility is shown in Figure 6 below. In this system, the diversionary type of fallacy can be a subspecies wrong conclusion and red herring.

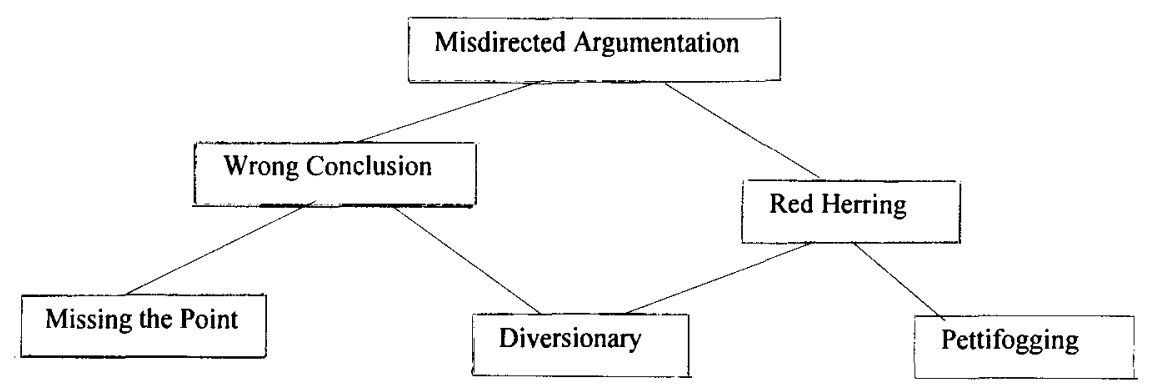

Figure 6: System 1 for Classification of Fallacies of Relevance

According to this system, the coffee and light bulb examples, to cite the most difficult cases to classify, would go under the diversionary category. Such cases would be classified as both wrong conclusion and red herring, on the grounds that they exhibit both a wrong conclusion and a strategic diversion. This is certainly one solution to the problem that should be considered. But as noted above, it is a difficult one to swallow. For the users of the system now have to be taught that many common examples of the kind they will confront in real cases may need to be put in the category of being both instances of the wrong conclusion fallacy and the red herring fallacy. On the other hand, this may be the best way to go, to do justice to the reality of the examples themselves. Maybe in real cases, the two modes of fallacious argumentation are really combined. Thus there seems to be a kind of dilemma involved in opting for System 1 or rejecting it. Is there an alternative system that could be considered?

Recall the cautionary note added at the end of the last section. In these borderline cases, the proposition supposed to represent the wrong conclusion had to be added in as an implicit conclusion in the argument. This suggests that in such a 
case it may be a little too strong to classify the fallacy committed as one of wrong conclusion. The reader will also recall that the critical questions for responding to cases of wrong conclusion seem to be different from those appropriate for responding to cases of red herring. This suggests that in cases like the coffee example and the pesticides example, it may be too strong to classify the fallacy as one of wrong conclusion. In such cases, the appropriate critical question should be, 'Is this your final conclusion?' What is suggested is an approach in which the critic should examine each case carefully on the evidence presented. If there is no wrong conclusion that can definitely be pinned down without any doubts about alternative interpretations of the argument, and there is evidence of strategic deception, the case could be classified as a red herring. Then a cautionary note could be added that there is another interpretation of the argument whereby it could be classified as an instance of wrong conclusion.

This approach could be based on a different way of applying the criteria for the two fallacies, using the following pair of heuristic guidelines:

(HWC) If the argumentation has definitely arrived at a wrong conclusion, classify it as wrong conclusion.

(HRH) If there is some doubt about whether the argumentation has arrived at a definitely wrong conclusion, and if there is strong evidence of strategic diversion, classify it as red herring.

Applying these guidelines is based on the point that the wrong conclusion is the more serious of the two fallacies and the easier to pin down by the evidence offered in a case. Thus if there is definite evidence that this fallacy has been committed, the critic should "go for it", and deploy this criticism. If, however, there is some evidence of a wrong conclusion, but this evidence is less than definite to pin down, but there is evidence of strategic diversion, then the critic should go with this lesser charge. The lesser charge involves the critic only asking where the argument is going, as opposed to accusing it of having gone to the wrong conclusion.

In line with this latter approach, wrong conclusion and red herring can be classified as two distinct fallacies, both falling under the more general category of misdirected argumentation. To appreciate this classification proposal, look back once more to the structure of argumentation in fallacies of irrelevance represented in Figure 1. If the line of argumentation first appears to go in the direction of the proper path towards the conclusion to be proved, but then later veers off towards a different or "wrong" conclusion, the fallacy is one of misdirected argumentation. If the argument actually arrives at this different conclusion, the fallacy is that of wrong conclusion, identified by the four criteria for this fallacy specified above. 
However, if it goes off the proper path, and towards a wrong conclusion, but doesn't actually arrive at it, the fallacy of irrelevance can be classified as a red herring. The evidence for this classification, indicated by the three criteria above, includes strategic diversion and failure to throw probative weight on the conclusion to be proved. Missing the point can then be classified as special subspecies of wrong conclusion, while diversionary irrelevance and pettifogging irrelevance can be classified as subspecies of the red herring fallacy.

In System 2, the diversionary subspecies comes only under red herring, and not under wrong conclusion as well. System 2 of classification is more consistent with the one offered in (Walton, 2004) than System 1. The difference between System 2 and the system proposed in (Walton, 2004, p. 243) is that missing the point has been added as a subspecies of wrong conclusion. A key difference is that in a case where the red herring fallacy has been committed, there is a strategic component exhibited when the arguer deliberately interjects with some colorful controversy that is meant to distract the audience by leading off on to this different issue. The strategy is meant to provide a diversion that will make the audience overlook the failure to prove the proper conclusion. But in a case of red herring, there is no definitely indicated wrong conclusion to which the argumentation is directed. Actually applying the distinction to real examples using these criteria, however, has to be built on an analysis of the argumentation in the individual case. A careful examination of the text of discourse must be made, including an assessment of what has been explicitly said and what has been left unsaid. Contextual factors of the dialogue in a case are important in making such a classification in any individual case, as the examples above have so well illustrated. Classifying longer cases would, in one respect anyhow, be easier, because more textual information is given, and therefore fewer speculative assumptions need to be made about what might have been said but not reported in the given text of the case. In dealing with any example however, one must operate on the following epistemic closure assumption: if no evidence was given that something was said, or that some digression was made, then for purposes of analysis and classification of any putative fallacy in the case, one must assume by default that for all we know, it was not said.

On this basis, the recommendation is that System 2 is better. Yet it seems likely that more examples will need to be analyzed before either system can be conclusively accepted or rejected. A nice feature of the approach System 2 is based on is that it classifies and evaluates any individual criticism that a fallacy has been committed in an argument in light of the critical questioning process that should be appropriate for the case.

A final comment is that classifying pure fallacies of relevance is a special problem. In many cases of classification of fallacies, the fallacy is based on an argument that has somehow been used wrongly, or is deficient in some respect. Thus the classification of fallacies (incorrect arguments) needs to be based on an underlying 
classification and analysis of argumentation schemes (correct forms of argumentation). Classification of pure fallacies represents a different sort of problem. Many argumentation schemes can be used, and what we are dealing with is a chain of argumentation that can be made up of many subarguments of various kinds. The fallacy arises not through the misuse of any single argumentation scheme, but through how the actual line of argumentation in a case moves towards or away from the proper line of argumentation that should have as its end point the ultimate conclusion to be proved. Thus, as has been shown above, utilization of a system of argument diagramming like Araucaria is vital to diagnose pure fallacies of relevance. The next step in this research is to re-examine other particular fallacies also classified as fallacies of relevance, like ad hominem, ad baculum, ad misericordiam, appeal to pity, and so forth, to see how they fit into the new classification system.

\section{Notes}

I The author would like to thank the Social Sciences and Humanities Research Council of Canada for a research grant "Argumentation Schemes in Natural and Artificial Communication" that supported the work in this paper, and to thank David Godden, Fabrizio Macagno and Chris Reed for discussions.

${ }^{2}$ Araucaria was used to analyze some examples of relevance in (Walton, 2004, 119-120, 183185), but not the examples studied in the present paper.

${ }^{3}$ A survey of textbook treatments of fallacies of irrelevance has been presented in (Walton, 2004, chapter 3 ), along with a general theory of relevance in argumentation.

${ }^{4}$ The method used to determine relevance of an argument in a given case in (Walton, 2004) is called argument extrapolation. Extrapolation is chaining forward of argumentation found in an existing text of discourse to see whether it moves toward or reaches an ultimate conclusion.

${ }^{5}$ For an account of Aristotle's view of misconception of refutation as a fallacy, see (Walton, 2004, 32-35).

${ }^{6}$ A detailed case study analyzing legal evidence in criminal case using argument diagramming has been presented in (Prakken, Reed and Walton, 2003). First the Wigmore chart method is used, and then the argumentation in the same case is analyzed using Araucaria.

${ }^{7}$ In the 1994 edition (p. 127), the first sentence of the light bulb example is, "The Consumers Digest reports that Sylvania light bulbs last longer than GE bulbs". The example makes more sense if the two light bulb manufacturers names are reversed, and so I have presented the light bulb example this way.

${ }^{8}$ This dead weight hypothesis was suggested by a comment made to me by Fabrizio Macagno after a lecture I gave on fallacies of relevance on January 8, 2004.

\section{References}

Copi, Irving M. and Carl Cohen. (1994). Introduction to Logic. (9th ed.). New York: Macmillan.

Copi, Irving M. and Carl Cohen. (2004). Essentials of Logic. Upper Saddle River, NJ: Pearson/Prentice Hall.

Hurley, Patrick J. (1994). A Concise Introduction to Logic. (5th ed.). Belmont, CA: Wadsworth. 


\section{Classification of Fallacies of Relevance 103}

Hurley, Patrick J. (2003). A Concise Introduction to Logic. (8th ed.). Belmont, CA: Wadsworth.

Prakken, Henry, Chris Reed and Douglas Walton. (2003). "Argumentation Schemes and Generalizations in Reasoning about Evidence," in Proceedings of the 9th International Conference on Artificial Intelligence and Law, Edinburgh, 2003, New York: ACM Press. Pp. 32-41. Available at http://www.uwinnipeg.ca/ walton

Chris Reed and Glenn Rowe. (2002). "Araucaria: Software for Puzzles in Argument Diagramming and XML," in Technical Report. Department of Applied Computing, University of Dundee. Available at http://www.computing.dundee.ac.uk/staff/creed/ araucaria/.

Reed, Chris and Glenn Rowe. (2003). Araucaria, Version 2, User Manual. Available at http://www.computing.dundee.ac.uk/staff/creed/araucaria/.

Walton, Douglas. (1995). A Pragmatic Theory of Fallacy. Tuscaloosa: University of Alabama Press.

Walton, Douglas. (2004). Relevance in Argumentation. Mahwah, NJ: Lawrence Erlbaum Associates.

Whately, Richard. (1870). Elements of Logic. (9th ed.). London: Longmans, Green Reader and Dyer.

Douglas Walton

Department of Philosophy

University of Winnipeg

515 Portage Avenue

Winnipeg, Manitoba

Canada R3B 2E9

d.walton@uwinnipeg.ca 